Canadian

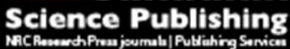

Applied Physiology, Nutrition, and Metabolism Physiologie appliquée, nutrition et métabolisme

\title{
A single Mediterranean meal does not impair postprandial flow-mediated dilatation in healthy men with sub-clinical metabolic dysregulations
}

\begin{tabular}{|r|l|}
\hline Journal: & Applied Physiology, Nutrition, and Metabolism \\
\hline Manuscript ID & apnm-2015-0490.R2 \\
\hline Manuscript Type: & Article \\
\hline Date Submitted by the Author: & $23-$ Feb-2016 \\
\hline Complete List of Authors: & $\begin{array}{l}\text { Lacroix, Sébastien; Montreal Heart institute, EPIC } \\
\text { Des Rosiers, Christine; Montreal Heart institute } \\
\text { Gayda, Mathieu; Montreal Heart institute, EPIC } \\
\text { Nozza, Anna; Montreal Heart institute, Coordonating Center } \\
\text { Thorin, Éric; Montreal Heart institute } \\
\text { Tardif, Jean-Claude; Montreal Heart institute } \\
\text { Nigam, Anil; Montreal Heart institute, EPIC }\end{array}$ \\
\hline Keyword: & $\begin{array}{l}\text { Endothelial function, plasma fatty acid composition, inflammation, } \\
\text { oxidative stress, cardiovascular disease risk factors }\end{array}$ \\
\hline
\end{tabular}




\section{A single Mediterranean meal does not impair postprandial flow-mediated dilatation in healthy men with sub-clinical metabolic dysregulations}

Sébastien Lacroix PhD ${ }^{1-3}$, Christine Des Rosiers PhD ${ }^{1-3}$, Mathieu Gayda PhD ${ }^{1,2,4}$, Anna Nozza MSc ${ }^{5}$, Éric Thorin PhD ${ }^{2,6}$, Jean-Claude Tardif MD $^{2,4}$ and Anil Nigam MD MSc ${ }^{1-4}$

${ }^{1}$ Cardiovascular Prevention and Rehabilitation Centre, Montreal Heart Institute, 5055 StZotique Street east, Montreal, Quebec, Canada, H1T 1 N6.

${ }^{2}$ Montreal Heart Institute Research center, 5000 Belanger Street, Montreal, Quebec, Canada, H1T $1 \mathrm{C} 8$.

${ }^{3}$ Department of Nutrition, Faculty of Medicine, Université de Montréal, 2405, Côte-SainteCatherine road, Montréal, Québec, Canada, H3T 1A8

${ }^{4}$ Department of Medicine, Université de Montréal, 2900, Édouard-Montpetit blv., Montréal, Québec, Canada, H3T 1J4

${ }^{5}$ Montreal Heart Institute Coordinating Center, 5000 Belanger Street, Montreal, Quebec, Canada, H1T 1 C8.

${ }^{6}$ Department of Surgery, Université de Montréal, 2900, Édouard-Montpetit blv., Montréal, Québec, Canada, H3T 1J4

Corresponding author: Dr. Anil Nigam; Montreal Heart Institute, 5000 Belanger Street, Montreal, Quebec, Canada, H1T 1C8. Telephone: (514) 376-3330; Fax (514) 374-2416; Email: anil.nigam@icm-mhi.org 


\section{ABSTRACT}

Introduction: Cardiovascular risk factors are known to exacerbate high-saturated fatty acid meal (HSFAM)-induced endothelial dysfunction but the influence of sub-clinical metabolic dysregulations and the acute impact of a single mixed Mediterranean-type meal (MMM) remains unknown and could especially benefit such individuals. Thus, this study has the objective to evaluate the metabolic and vascular effect of such meals in healthy subjects with or without sub-clinical fasting metabolic dysregulations.

Material and Methods: Twenty-eight (28) healthy males without overt cardiovascular risk factors randomly ingested one of two isocaloric meals on separate days. Plasma metabolic markers, fatty acid (FA) profile and endothelial function (Flow-mediated dilatation; FMD) were assessed at baseline and 2 and 4 hours after meal ingestion. Unsupervised hierarchical clustering identified two subgroups of participants $(n=11$ and 17$)$ differing by their baseline metabolic profiles.

Results: The MMM did not significantly alter postprandial endothelial function in all subjects, irrespective of baseline metabolic parameters. In contrast, the HSFAM induced postprandial endothelial dysfunction $\left(\Delta \% \mathrm{FMD}_{\text {absolute }}=-5.28 \pm 2.54\right.$, $\mathrm{p}$-value $<0.01 \mathrm{vs}$. MMM) in a subgroup of individuals with significantly greater BMI, fasting insulinemia and lipid parameters $(n=11)$. Finally, the postprandial plasma FA profiles were differentially enriched by the HSFAM and MMM, notably with saturated FAs and omega-3 polyunsaturated FAs, respectively.

Conclusions: Collectively, our results highlight the detrimental impact of a single HSFAM on endothelial function in healthy individuals displaying sub-clinical fasting metabolic dysregulations. Such individuals could benefit from MMM, demonstrated herein to be without any acute detriment to endothelial function.

Keywords: Endothelial function, plasma fatty acid composition, inflammation, oxidative stress, cardiovascular disease risk factors 


\section{RÉSUMÉ}

Introduction: Les facteurs de risque cardiovasculaire sont reconnus pour exacerber la dysfonction endothéliale suivant la consommation de repas riche en acides gras saturés (HSFAM). Cependant, l'impact de dérèglements métaboliques sous-cliniques demeure méconnu comme l'est l'effet d'un repas de type méditerranéen (MMM). Cette étude a donc pour objectif d'évaluer les réponses métabolique et endothéliale à de tels repas chez des sujets sains avec ou sans dérèglement métabolique sous-clinique.

Matériel et Méthodes : Vingt-huit (28) hommes sains, sans facteurs de risque cardiovasculaires ont reçu de façon randomisée un des repas à l'étude. Des marqueurs métaboliques et le profil d'acides gras (AG) plasmatiques, ainsi que la fonction endothéliale (Flow-mediated dilatation; FMD) ont été évalués à jeun et 2 et 4 heures postprandiales. Un algorithme de regroupement hiérarchique non-supervisé a identifié deux sous-groupes de participants ( $\mathrm{n}=11$ and 17 ) sur la base de certains paramètres métaboliques à jeun.

Résultats : Aucune altération endothéliale postprandiale significative n'a été observée suivant le MMM. Le HSFAM a induit une dysfonction endothéliale postprandiale $\left(\Delta \% \mathrm{FMD}_{\text {absolue }}=-5.28 \pm 2.54 \mathrm{p}\right.$-value $<0.01$ vs. $\left.\mathrm{MMM}\right)$ seulement chez les individus à l'IMC, l'insulinémie et aux paramètres lipidiques à jeun plus élevés $(n=11)$. Finalement, le profil d'AGs postprandial a été enrichi différemment par le HSFAM et le MMM, notamment en AGs saturés et polyinsaturés oméga-3, respectivement.

Conclusions: Nos résultats démontrent l'impact négatif d'un HSFAM sur la fonction endothéliale postprandiale de sujets sains avec dérèglements métaboliques sous-cliniques. Ces individus pourraient bénéficier de la consommation de MMM, démontré ici pour ne pas altérer la fonction endothéliale.

Mots-clés : Fonction endothéliale, composition d'acides gras plasmatiques, inflammation, stress oxydatif, facteurs de risque de maladie cardiovasculaire 


\section{INTRODUCTION}

Cardiovascular and metabolic diseases are a growing global burden (Chen et al. 2012; Mozaffarian et al. 2015) as is the industrialization of the food supply and the westernization of daily habits. In fact, in the past few decades, consumption of sodium, refined sugars, saturated fatty acids (SFA), as well as the proportion of omega- 6 vs. omega3 polyunsaturated fatty acids (PUFA) has increased (Carrera Bastos 2011) and is at least partly to blame for the deterioration of global cardiometabolic health. In contrast, increased adherence to a Mediterranean-type diet was shown to improve cardiometabolic health and prevent cardiovascular events (Estruch et al. 2013; Sofi et al. 2014).

Another hallmark of recent westernization of habits is increasing periods of time spent in the postprandial state, which was shown to be associated with detrimental vascular consequences (Cordain et al. 2005; Lopez-Miranda et al. 2007). One notable manifestation of repeated postprandial insults is the impairment of endothelial function, a well-established step in atherogenesis initiation (Zilversmit 1979; Bonetti et al. 2003). Indeed, endothelial dysfunction alone is predictive of future cardiovascular events in healthy individuals and in patients with established cardiovascular disease (Witte 2005; Inaba et al. 2010; Matsuzawa et al. 2015). In this regard, the investigation of the body's ability to restore homeostasis in the few hours following physiological stressors such as meal ingestion can help identify early metabolic defects that would otherwise not be captured during the fasting state (van Ommen et al. 2009). The first demonstration by Vogel et al. (Vogel et al. 1997) of postprandial impairments in metabolic and endothelial functions following a single high-SFA meal (HSFAM) has since been corroborated on multiple occasions and is more frequently observed in individuals with cardiovascular risk factors (Lacroix et al. 2012). Meal fatty acid (FA) composition and/or antioxidant content would appear to influence the whole-meal effect on endothelial function. The addition of single elements from the cardioprotective Mediterranean diet such as olive oil, fish or fish oil was previously shown to attenuate the 
deleterious effects of a HSFAM on vascular reactivity (Plotnick et al. 1997; Vogel et al. 2000; Fahs et al. 2010; Lacroix et al. 2012). Most of these studies were, however, conducted with high and unrealistic quantities of isolated Mediterranean ingredients, thereby disregarding the potential synergy between multiple constituents of the Mediterranean diet.

Hence, the objective of the present study was to assess the acute impact of a complete mixed Mediterranean-type meal (MMM) vs. HSFAM on endothelial and metabolic functions in healthy males with or without sub-clinical fasting metabolic dysregulations.

\section{MATERIAL AND METHODS}

\section{Study population}

Twenty-eight male subjects, aged 18 to 50 years and free of cardiovascular disease or risk factors were recruited on a self-referral basis from the cardiovascular prevention and research centres of the Montreal Heart Institute. Potential participants were screened on the basis of their medical and familial history, medication use and blood work. Exclusion criteria were known/documented vascular disease (coronary, cerebral or peripheral), heart failure, left ventricular ejection fraction $<40 \%$, renal insufficiency (creatinine clearance $<90 \mathrm{ml} / \mathrm{min}$ ), hypertension (blood pressure $>140 / 90 \mathrm{mmHg}$ or treated), dyslipidemia (LDL-C $>3.5$ $\mathrm{mmol} / \mathrm{L})$, diabetes mellitus $(\mathrm{Hb} 1 \mathrm{Ac}>6.5 \%$ or treated), abdominal obesity (waist circumference $>102 \mathrm{~cm}$ ), acute infection, any medication use, nitroglycerin intolerance, history of alcohol abuse, smoking (active or within the past year) and supplementation with omega-3 PUFA (past 3 months) or vitamin C (past 2 weeks). This study was conducted according to the guidelines laid down in the Declaration of Helsinki and the Montreal Heart Institute ethics committee approved all procedures. Written informed consent was obtained from all subjects.

Intervention 
Study participants were randomly assigned to two different meal sequences using a crossover design (MMM - HSFAM or HSFAM - MMM). Both isocaloric test meals were separated by a minimum of 7 and a maximum of 30 days. The MMM consisted of fresh salmon with onion and almond pesto served with green beans, cherry tomatoes and potatoes all baked in extra-virgin olive oil (EVOO). Although salmon does not occur naturally in the Mediterranean sea, we refer to our MMM as a Mediterranean-type meal due to its nutritional composition (rich in omega-3 PUFAs, oleic acid, polyphenols and other antioxidant content) consistent with a Mediterranean-type diet, and the use of other ingredients that are typical of such a diet (EVOO, almonds, beans and tomatoes). The HSFAM consisted of a sausage, egg and cheese breakfast sandwich with three servings of hash brown potatoes obtained from a multinational fast-food chain on the day of each evaluation. The nutritional composition of both test meals is listed in Table 1. Nutritional compositions of both meals were obtained from the Food Processor Nutrition Analysis software (esha research, USA). Content of most macro- and micronutrients was similar between meals with the exception of SFA, omega-3 PUFA, cholesterol, fibres, and vitamin $\mathrm{C}$, which are inherent differences between the Mediterranean and Western-type diets and their representative meals. Meals were consumed in the early morning (9:00 - 9:30 am) after an overnight fast. Subjects were asked to refrain from strenuous exercise and alcohol consumption for 24 hours prior to both study visits. Medical personnel performing evaluations were blinded to intervention sequence.

\section{Anthropometric measures and blood samples}

Subjects underwent a medical history, physical examination with measurement of height, weight and body composition with tetrapolar bio-electrical impedance (Tanita BC418, Tanita Corporation, Japan). Blood was drawn in the fasted state (Baseline) and at two (T2) and four (T4) hours into the postprandial period (i.e. following meal intake). These time points were chosen since meals rich in fatty acids elicit most of their effect between 2 and 4 hours into the postprandial period (Vogel et al. 1997). Quantifications of blood glucose, 
insulin, lipid profile and high-sensitivity C-reactive protein (hsCRP) were performed by a Siemens Dimension RxL (Siemens AG, Germany). LDL cholesterol levels were calculated with the Friedewald formula, which is highly dependant on triglyceride levels. For that reason, postprandial LDL levels will be provided but not further interpreted. High sensitivity IL-6 (hsIL6) and myeloperoxidase (MPO) were evaluated by immunoassays (R\&D Systems HS600B and Alpco Diagnostics 30-6631A, respectively). Of note, hsIL6 and MPO were quantified only in a subset of six individuals and served as an exploratory investigation.

Background dietary habits, and more specifically those related to fatty acid intake, were evaluated using a validated 14-item food frequency questionnaire (FFQ) for cardiovascular prevention that results in a score reflecting global dietary pattern and sub-scores reflecting SFA, MUFA, omega-3 PUFA, omega-6 to omega-3 PUFA ratio and fruit and vegetable intakes (Laviolle et al. 2005). This questionnaire was recently shown to appropriately estimate the erythrocyte levels of vegetable and marine sources of omega-3 PUFAs, and of omega-6 PUFAs (Turcot et al. 2015). Furthermore, past and present physical activity habits were assessed using a validated 10 -item questionnaire that estimates $\mathrm{VO}_{2}$ max levels with very high reliability (Trivel et al. 2004).

\section{Endothelium-dependant dilatation}

Endothelial function was evaluated in the fasted state and 2 and 4 hours following ingestion of the test meal (T2 and T4, respectively). Brachial ultrasonography was performed according to published guidelines (Corretti et al. 2002) following a 5-minute brachial artery occlusion using a pressure cuff connected to an automated cuff inflator (D. E. Hokanson Inc., model E20, USA) positioned above the right elbow and inflated at $80 \mathrm{mmHg}$ over the systolic blood pressure. Images were analyzed offline using the Brachial analyzer for Research software (Medical Imaging Applications LLC, USA) by a single technician blinded to the meal sequence in order to minimize variability and potential bias. Flow-mediated dilatation (FMD) was defined as the maximal percent of arterial dilatation from baseline 
during post-occlusive reactive hyperemia. Using this method, the intra- and inter-observer variability for brachial artery diameter determinations are $0.056 \pm 0.024$ and $0.073 \pm 0.031$ $\mathrm{mm}$, respectively (Nigam et al. 2003). The intra-subject coefficient of variation between fasting FMD measurements was $9 \%$, which is lower than previously published values (Herrington et al. 2001). Resting and hyperemic blood flow was also measured at rest and following cuff deflation.

\section{Endothelium-independent dilatation}

Fasting nitroglycerin-mediated dilatation (NMD), a marker of endothelium-independent dilatation, was assessed five minutes after completion of the evaluation of FMD according to published guidelines (Corretti et al. 2002). Nitroglycerin-mediated dilatation was defined as the maximal percent of arterial dilatation during a 3-minute period following administration of a sublingual vaporisation of $0.4 \mathrm{mg}$ of nitroglycerin. Endothelium-independent dilatation was evaluated to ensure normal vascular smooth muscle vasomotor function.

\section{Total plasma fatty acid (FA) profile}

Total fasting and postprandial (T4) plasma FAs, free and bound to triglycerides and phospholipids, were characterised by gas chromatography coupled to mass spectroscopy following a previously described methodology (Gelinas et al. 2011). Individual FAs were expressed as a percentage of total plasma FA concentration and thus represent a proportion of total plasma FAs.

\section{Statistical analysis}

The study population size of 28 subjects provided $90 \%$ power to detect a $2 \%$ absolute variation in FMD, which was previously shown to be clinically significant (Corretti et al. 2002; Nigam et al. 2003) and to be associated to adjusted risk of future cardiovascular events (Matsuzawa et al. 2015). A post-hoc unsupervised agglomerative hierarchical clustering (hclust R function) analysis identified two subgroups of participants based on the similarity of 
their baseline characteristics (age, BMI, glucose, insulin, HOMA-IR, Total, LDL and HDL cholesterol, triglycerides and cholesterol / HDL). Shapiro-Wilk test was used to evaluate normality of distribution and data not following normality were log-transformed before analysis or analysed with non-parametric tests such as the case of plasma FA. No evidence of meal sequence carry-over effect (two-way ANOVA using meal sequence as interaction term) and no significant differences in fasting (baseline) parameters between study visits were observed. Baseline parameters were thus averaged to simplify data presentation. Postprandial area under the curve (AUC) was calculated according to the trapezoid method (pracma R package). All tests were two-sided and conducted at the 0.05 significance level. Normally distributed values are presented as mean $\pm S D$, while those not following normality are presented as median [Q1, Q3]. Analyses were performed with the R software version 3.0.1 (R Development Core Team 2011).

\section{RESULTS}

Averaged baseline characteristics for both study visits are shown in Table 2 and are reflective of a healthy cohort without evidence of cardiovascular risk factors and with normal fasting endothelial function (i.e. FMD of $10.7 \pm 2.8 \%$ ). Moreover, the average total plasma FA content was $1,329[1,079,1,570] \mathrm{nmol} / \mathrm{L}$.

Identification of metabolic subgroups and baseline characteristics

An unsupervised hierarchical clustering algorithm defined two subgroups of participants based on the similarity between the baseline characteristics detailed in Table 2 . The subgroups generated comprised 11 (group 1) and 17 (group 2) individuals, respectively. Post-hoc power calculations revealed that the sample sizes of the aforementioned subgroups provided $80 \%$ power to detect a $2.5 \%$ and a $2 \%$ absolute variation in FMD, respectively. Group 1 (also referred to as the dysmetabolic group) was composed of subjects with significantly higher BMI, fasting insulin and HOMA-IR, fasting total and non- 
HDL cholesterol and fasting triglycerides and triglyceride to HDL ratio in comparison to subjects from group 2 (healthy group) ( $p$-value $<0.05$ vs. group 2) (Table 2). Moreover, total body fat and trunk fat percentages tended to be higher in group 1 ( $p$-value $<0.1$ vs. group 2). Subjects within the dysmetabolic group (group 1) also had significantly lower HDL cholesterol ( $p$-value < 0.01 vs. group 2, MannW). Importantly, fasting endothelial parameters (\%FMD, resting arterial diameter and \%NMD) were not significantly different between subgroups. Finally, baseline total FA levels were significantly greater in dysmetabolic subjects (group 1) than those within group $2(1,585[1,437,1,885]$ vs. $1,188[1,053,1,355]$ $\mathrm{nmol} / \mathrm{L}$ respectively, $\mathrm{p}$-value $<0.05 \mathrm{MannW})$.

As for the dietary habits and fitness levels, most of the FFQ items were not significantly different between groups 1 and 2 with the exception of daily fruit and vegetable consumption, which was significantly lower for dysmetabolic individuals (group 1) (p-value $<0.05$, MannW) (Table 3). Fitness levels (estimated VO2max) were not significantly different and within normal age-adjusted ranges for all individuals (Table 3).

\section{Postprandial metabolic and endothelial responses to test meals}

The ingestion of both test meals resulted in significant elevations in insulin and triglycerides in both groups (Figure $1 \mathrm{~A}$ and B and Supplementary table S1). Meal composition influenced the amplitude of the hypertriglyceridemic response solely in group 2 ( $p$-value $<0.005$ Wilcoxon between meals). Group 2 also experienced a less significant increase in triglycerides following the MMM in comparison to group 1 ( $p$-value $<0.05$ MannW between groups). Area under the triglyceride curve $\left(\mathrm{AUC}_{\mathrm{TG}}\right)$ was significantly higher in dysmetabolic subjects (group 1) (p-value < 0.05 vs. group 2, MannW) and was higher following the HSFAM within group 2 ( $p$-value $<0.05$ vs. MMM, Wilcoxon). Finally, no significant postprandial variations in hsCRP, hsIL6 or MPO were observed (Supplementary table S2). 
The HSFAM induced significant postprandial endothelial dysfunction 4 hours following meal ingestion only in dysmetabolic individuals (group 1) $\left(\Delta \% \mathrm{FMD}_{\text {absolute }}=-5.28 \pm 2.54 \mathrm{p}\right.$-value $<$ 0.01 vs. MMM and p-value < 0.01 vs. group 2), while the MMM had no significant effect on postprandial endothelial function in either group (Figure 2 and Supplementary table S1). Additionally, the $95 \%$ confidence interval of the observed 4-hour postprandial FMD following the HSFAM in group 1 individuals (i.e $95 \% \mathrm{Cl}$ from 4.34 to $7.34 \% \mathrm{FMD}$ ) does not exceed their baseline FMD level. This adds robustness to the postprandial FMD alterations observed in these dysmetabolic individuals.

Plasma fatty acid profiles following test meals

No significant group differences in postprandial variations in FA profile to meals were observed. Postprandial FAs results are therefore presented for the entire cohort $(n=28)$. Both meals induced postprandial total FA elevation that was significantly greater following the $\operatorname{HSFAM}(\Delta$ Total FA $\mathrm{HSFAM}=295[130,519] \mathrm{nmol} / \mathrm{L})$ than the MMM $\left(\Delta\right.$ Total $_{\mathrm{FA}} \mathrm{A}_{\mathrm{MMM}}=139[26$, 333] $\mathrm{nmol} / \mathrm{L}, \mathrm{p}$-value $=0.01$ between meals). Both test meals also induced variation in postprandial FA composition (Figure 3 and Supplementary table S3). For instance, the proportions of SFA (C16:0), MUFA (C18:1n9, C20:4), omega-6 PUFA (C18:2n6) and each omega-3 PUFA were significantly modified in the postprandial state by both meals ( $p$-value < 0.05 vs. baseline). Furthermore, the postprandial proportions of several FAs, namely C18:0, C18:1n9, 18:2n6 and each omega-3 PUFA, were significantly influenced by meal composition ( $p$-value $<0.05$ between meals). Notably, postprandial plasma omega-3 to omega-6 PUFA ratios were significantly increased only following the MMM ( $\Delta$ omega3:omega- $6=0.006[0.001,0.02]$ vs. $-0.002[-0.007,0.003]$ following the HSFAM, p-value < $0.001)$.

\section{DISCUSSION}


This study is, to our knowledge, the first to have 1- investigated the influence of a single and complete meal reflective of a Mediterranean-type diet on postprandial endothelial function, 2- concomitantly evaluated postprandial circulating FA composition, and 3investigated the impact of sub-clinical metabolic dysregulations on these parameters. Our results demonstrate for the first time that a MMM does not alter postprandial endothelial function in healthy males, regardless of the fasting metabolic profile. The fact that the MMM is less challenging for postprandial endothelial homeostasis adds to the many characteristics of the Mediterranean-type diet and presumably contributes to its cardiometabolic benefits. Interestingly, we also observed that an isocaloric HSFAM impairs postprandial endothelial function, but only in a subgroup of healthy individuals with sub-clinical metabolic dysregulations.

The neutral effect on endothelial function exhibited by the MMM in our cohort could be attributable to, among other factors, its EVOO content known for its favorable fatty acid content and anti-inflammatory and antioxidant polyphenol composition (Ghanim et al. 2011). The addition of EVOO to fat-rich meals was shown to improve postprandial glucose, LDL and oxidized-LDL and to prevent increases in inflammation, endothelial adhesion molecules and oxidative stress associated with consumption in healthy and hypertriglyceridemic subjects (Bogani et al. 2007; Pacheco et al. 2008; Violi et al. 2015). In the present study, MMM ingestion in fact resulted in plasma oleic acid $(18: 1 \mathrm{n} 9)$ elevation that could have acted in synergy with polyphenols to explain the neutrality of the MMM on endothelial function. Furthermore, ingestion of the MMM, which was also an important source of long-chain omega-3 PUFAs, resulted in significant increases in their circulating levels and of the omega-3 to omega-6 PUFA ratio. The acute elevation of this class of PUFA is known to result in the production of eicosanoids and oxylipins with lesser inflammatory potential than those synthesized from omega-6 PUFA and SFA (Armah et al. 2008; Margioris 2009; Strassburg et al. 2014) and to attenuate HSFAM-induced endothelial impairments (Fahs et 
al. 2010). Altogether, these features contribute to decreasing the stress induced by the MMM on metabolic and vascular homeostatic mechanisms.

The impairments in endothelial function that occurred following the HSFAM in dysmetabolic subjects (group 1) are also of interest since they corroborate what was previously observed in a cohort of subjects with high cardiometabolic risk factors. In fact, most trials reporting a significant impairment of postprandial endothelial function following a HSFAM did so in participants with overt cardiovascular risk factors such as hypertriglyceridemia (Giannattasio et al. 2005; Norata et al. 2006), abdominal obesity (Wang et al. 2011), and/or type 2 diabetes mellitus (West et al. 2005). Recently, healthy subjects with elevated anthropometric and fasting metabolic parameters were found to exhibit metabolic, inflammatory and endocrine responses to a high-SFA beverage that were intermediary between those of healthier subjects and subjects with metabolic syndrome (Kardinaal et al. 2015). In the present trial, those healthy individuals that displayed postprandial endothelial impairments following the HSFAM (group 1) had adiposity, fasting insulinemia and lipemia (notably triglyceridemia), which were significantly higher than their counterparts (group 2), although all values remained within ranges regarded as "normal".

The aforementioned marginally altered anthropometric and fasting metabolic parameters in otherwise healthy individuals could thus exacerbate the postprandial endothelial - and metabolic - sensitivity to meal composition and be early indicators of increased risk of postprandial impairments. In fact, the sub-clinical metabolic alterations exhibited by group 1 seem to have increased their susceptibility to the unfavourable composition of the HSFAM (i.e. high SFA and cholesterol content, low omega-3 to omega-6 PUFA ratio, and low antioxidant content) in comparison to the MMM. For one, these phenotypes could impair the - or be the result of impaired - postprandial handling of the lipid load that inevitably arises from the ingestion of fat-rich meals. Indeed, dysmetabolic individuals (group 1) experienced a prolonged postprandial period spent under a greater triglyceridemic load (i.e. greater 
$A U C_{T G}$ than group 2), which in addition, was enriched in SFA like stearic acid (C18:0) following the HSFAM. Secondly, the marginal metabolic profile exhibited by group 1 might also have blunted their ability to handle postprandial inflammatory and/or oxidative insults. Incidentally, dysmetabolic individuals (group 1) reported a lower fruit and vegetable consumption, which could also have contributed in lowering their basal anti-inflammatory and antioxidant status. This item of the FFQ was in fact shown to correlate positively with plasma vitamin $\mathrm{C}$ and folate concentrations, both of which exert antioxidant effects (Laviolle et al. 2005).

Some elements of the present trial might be regarded as limitations to the interpretation and generalization of results. Firstly, the inclusion of participants was limited to healthy men below 50 years of age in an effort to minimize potential confounders such as the effect of aging and of the female hormonal cycle on endothelial function (Harris et al. 2012; Higashi et al. 2012) and it's evaluation by FMD (Witte 2005). Secondly, the quantification of inflammatory and oxidative stress biomarkers in a statistically insufficient subset of participants might have led to misinterpretation of the involvement of these processes in the observed postprandial endothelial events. This however does not eliminate the potential involvement of - low-grade - oxidative stress and/or inflammation processes at the systemic and/or vascular endothelial level (Wopereis et al. 2013). Elevation of postprandial plasma inflammation and/or oxidative stress are rarely and still inconsistently reported in healthy subjects (Lacroix et al. 2012) and should be interpreted with caution since they often only reflect local inflammatory response to venous cannulation, a procedure not performed here (Herieka et al. 2013; Wopereis et al. 2013). Finally, the choice of a short semi-quantitative FFQ aimed specifically at evaluating the quality of fatty acid intake may not have adequately taken into account the impact of habitual intake of nutrients other than fatty acids on endothelial responses to test meals.

\section{CONCLUSIONS}


Altogether, our observations suggest that sub-clinical metabolic dysregulations are early signs of increased endothelial and metabolic sensitivity to meal composition. Individuals with such a phenotype could thus be at increased risk for postprandial endothelial dysfunction following meals inducing a greater stress on homeostatic mechanisms, such as HSFAM. These individuals would thus benefit even more from consuming meals representative of a Mediterranean-type diet demonstrated herein for their non-deleterious endothelial properties. This study also further highlights the importance of challenging homeostasis to uncover otherwise unrecognized postprandial abnormalities, thus improving the evaluation of the cardiovascular risk and personalization of dietary recommendations.

Upon confirmation in a larger cohort of healthy individuals with marginally dysregulated fasting parameters, these findings ultimately will deepen the understanding of homeostatic mechanisms that maintain and determine vascular and overall health and will highlight the importance of the nutritional composition of each and every meal, even in seemingly healthy individuals.

\section{ACKNOWLEDGEMENTS}

The authors wish to acknowledge the assistance of study coordinator Julie Lalongé, echography technicians Danielle Gélinas and Marie Gagnon, the nursing staff of the Cardiovascular prevention and rehabilitation center of the Montreal Heart Institute, the director of the clinical biochemistry laboratory Dr. Joel Lavoie and Dr. Jean Lambert, senior biostatistician from the Montreal Heart Institute Coordinating Center.

\section{FUNDING SOURCES}


This study was funded by the Cardiovascular Prevention and Rehabilitation Centre foundation of the Montreal Heart Institute and the Montreal Heart Institute Foundation.

\section{DISCLOSURES}

The authors of this study have no relevant conflict of interest to disclose. 


\section{REFERENCES}

Armah, C.K., Jackson, K.G., Doman, I., James, L., Cheghani, F., andMinihane, A.M. 2008. Fish oil fatty acids improve postprandial vascular reactivity in healthy men. Clin Sci (Lond) 114(11): 679-86.

Bogani, P., Galli, C., Villa, M., andVisioli, F. 2007. Postprandial anti-inflammatory and antioxidant effects of extra virgin olive oil. Atherosclerosis 190(1): 181-6.

Bonetti, P.O., Lerman, L.O., andLerman, A. 2003. Endothelial dysfunction: a marker of atherosclerotic risk. Arterioscler Thromb Vasc Biol 23(2): 168-75.

Carrera Bastos, P., Fontes-Villalba M., O'Keefe JH., Lindeberg S. and Cordain L 2011. The western diet and lifestyle and diseases of civilization. Research Reporst in Clinical Cardiology 215-35.

Chen, L., Magliano, D.J., andZimmet, P.Z. 2012. The worldwide epidemiology of type 2 diabetes mellitus--present and future perspectives. Nat Rev Endocrinol 8(4): 228-36.

Cordain, L., Eaton, S.B., Sebastian, A., Mann, N., Lindeberg, S., Watkins, B.A., et al. 2005. Origins and evolution of the Western diet: health implications for the 21 st century. Am J Clin Nutr 81(2): 341-54.

Corretti, M.C., Anderson, T.J., Benjamin, E.J., Celermajer, D., Charbonneau, F., Creager, M.A., et al. 2002. Guidelines for the ultrasound assessment of endothelial-dependent flowmediated vasodilation of the brachial artery: a report of the International Brachial Artery Reactivity Task Force. J Am Coll Cardiol 39(2): 257-65.

Estruch, R., Ros, E., Salas-Salvado, J., Covas, M.I., Corella, D., Aros, F., et al. 2013. Primary prevention of cardiovascular disease with a Mediterranean diet. N Engl J Med 368(14): 1279-90.

Fahs, C.A., Yan, H., Ranadive, S., Rossow, L.M., Agiovlasitis, S., Wilund, K.R., et al. 2010. The effect of acute fish-oil supplementation on endothelial function and arterial stiffness following a high-fat meal. Appl Physiol Nutr Metab 35(3): 294-302. 
Gelinas, R., Thompson-Legault, J., Bouchard, B., Daneault, C., Mansour, A., Gillis, M.A., et al. 2011. Prolonged QT interval and lipid alterations beyond beta-oxidation in very long-chain acyl-CoA dehydrogenase null mouse hearts. Am J Physiol Heart Circ Physiol 301(3): H81323.

Ghanim, H., Sia, C.L., Korzeniewski, K., Lohano, T., Abuaysheh, S., Marumganti, A., et al. 2011. A resveratrol and polyphenol preparation suppresses oxidative and inflammatory stress response to a high-fat, high-carbohydrate meal. J Clin Endocrinol Metab 96(5): 140914.

Giannattasio, C., Zoppo, A., Gentile, G., Failla, M., Capra, A., Maggi, F.M., et al. 2005. Acute effect of high-fat meal on endothelial function in moderately dyslipidemic subjects.

Arterioscler Thromb Vasc Biol 25(2): 406-10.

Harris, R.A., Tedjasaputra, V., Zhao, J., andRichardson, R.S. 2012. Premenopausal women exhibit an inherent protection of endothelial function following a high-fat meal. Reprod Sci 19(2): $221-8$.

Herieka, M. and Erridge, C. 2013. High-fat meal induced postprandial inflammation. Mol Nutr Food Res.

Herrington, D.M., Fan, L., Drum, M., Riley, W.A., Pusser, B.E., Crouse, J.R., et al. 2001. Brachial flow-mediated vasodilator responses in population-based research: methods, reproducibility and effects of age, gender and baseline diameter. J Cardiovasc Risk 8(5): 319-28.

Higashi, Y., Kihara, Y., andNoma, K. 2012. Endothelial dysfunction and hypertension in aging. Hypertens Res.

Inaba, Y., Chen, J.A., andBergmann, S.R. 2010. Prediction of future cardiovascular outcomes by flow-mediated vasodilatation of brachial artery: a meta-analysis. Int $\mathrm{J}$ Cardiovasc Imaging 26(6): 631-40.

Kardinaal, A.F., van Erk, M.J., Dutman, A.E., Stroeve, J.H., van de Steeg, E., Bijlsma, S., et al. 2015. Quantifying phenotypic flexibility as the response to a high-fat challenge test in different states of metabolic health. FASEB J. 
Lacroix, S., DesRosiers, C., Tardif, J.C., andNigam, A. 2012. The role of oxidative stress in postprandial endothelial dysfunction. Nutr Res Rev 25(2): 288-301.

Laviolle, B., Froger-Bompas, C., Guillo, P., Sevestre, A., Letellier, C., Pouchard, M., et al. 2005. Relative validity and reproducibility of a 14-item semi-quantitative food frequency questionnaire for cardiovascular prevention. Eur J Cardiovasc Prev Rehabil 12(6): 587-95. Lopez-Miranda, J., Williams, C., andLairon, D. 2007. Dietary, physiological, genetic and pathological influences on postprandial lipid metabolism. Br J Nutr 98(3): 458-73.

Margioris, A.N. 2009. Fatty acids and postprandial inflammation. Curr Opin Clin Nutr Metab Care 12(2): 129-37.

Matsuzawa, Y., Kwon, T.G., Lennon, R.J., Lerman, L.O., andLerman, A. 2015. Prognostic Value of Flow-Mediated Vasodilation in Brachial Artery and Fingertip Artery for Cardiovascular Events: A Systematic Review and Meta-Analysis. J Am Heart Assoc 4(11). Mozaffarian, D., Benjamin, E.J., Go, A.S., Arnett, D.K., Blaha, M.J., Cushman, M., et al. 2015. Executive summary: heart disease and stroke statistics-2015 update: a report from the american heart association. Circulation 131(4): 434-41.

Nigam, A., Mitchell, G.F., Lambert, J., andTardif, J.C. 2003. Relation between conduit vessel stiffness (assessed by tonometry) and endothelial function (assessed by flow-mediated dilatation) in patients with and without coronary heart disease. Am J Cardiol 92(4): 395-9. Norata, G.D., Grigore, L., Raselli, S., Seccomandi, P.M., Hamsten, A., Maggi, F.M., et al. 2006. Triglyceride-rich lipoproteins from hypertriglyceridemic subjects induce a proinflammatory response in the endothelium: Molecular mechanisms and gene expression studies. J Mol Cell Cardiol 40(4): 484-94.

Pacheco, Y.M., Lopez, S., Bermudez, B., Abia, R., Villar, J., andMuriana, F.J. 2008. A meal rich in oleic acid beneficially modulates postprandial sICAM-1 and SVCAM-1 in normotensive and hypertensive hypertriglyceridemic subjects. J Nutr Biochem 19(3): 200-5.

Plotnick, G.D., Corretti, M.C., andVogel, R.A. 1997. Effect of antioxidant vitamins on the transient impairment of endothelium-dependent brachial artery vasoactivity following a single high-fat meal. JAMA 278(20): 1682-6. 
R Development Core Team 2011. R: A Language and Environment for Statistical Computing. Vienna, Austria : the R Foundation for Statistical Computing. R Foundation for Statistical Computing.

Sofi, F., Macchi, C., Abbate, R., Gensini, G.F., andCasini, A. 2014. Mediterranean diet and health status: an updated meta-analysis and a proposal for a literature-based adherence score. Public Health Nutr 17(12): 2769-82.

Strassburg, K., Esser, D., Vreeken, R.J., Hankemeier, T., Muller, M., van Duynhoven, J., et al. 2014. Postprandial fatty acid specific changes in circulating oxylipins in lean and obese men after high-fat challenge tests. Mol Nutr Food Res 58(3): 591-600.

Trivel, D., Calmels, P., Leger, L., Busso, T., Devillard, X., Castells, J., et al. 2004. Validity and reliability of the Huet questionnaire to assess maximal oxygen uptake. Can J Appl Physiol 29(5): 623-38.

Turcot, V., Brunet, J., Daneault, C., Tardif, J.C., Des Rosiers, C., andLettre, G. 2015. Validation of fatty acid intakes estimated by a food frequency questionnaire using erythrocyte fatty acid profiling in the Montreal Heart Institute Biobank. J Hum Nutr Diet 28(6): 646-58.

van Ommen, B., Keijer, J., Heil, S.G., andKaput, J. 2009. Challenging homeostasis to define biomarkers for nutrition related health. Mol Nutr Food Res 53(7): 795-804.

Violi, F., Loffredo, L., Pignatelli, P., Angelico, F., Bartimoccia, S., Nocella, C., et al. 2015. Extra virgin olive oil use is associated with improved post-prandial blood glucose and LDL cholesterol in healthy subjects. Nutr Diabetes 5e172.

Vogel, R.A., Corretti, M.C., andPlotnick, G.D. 1997. Effect of a single high-fat meal on endothelial function in healthy subjects. Am J Cardiol 79(3): 350-4.

Vogel, R.A., Corretti, M.C., andPlotnick, G.D. 2000. The postprandial effect of components of the Mediterranean diet on endothelial function. J Am Coll Cardiol 36(5): 1455-60.

Wang, Y.I., Schulze, J., Raymond, N., Tomita, T., Tam, K., Simon, S.I., et al. 2011. Endothelial inflammation correlates with subject triglycerides and waist size after a high-fat meal. Am J Physiol Heart Circ Physiol 300(3): H784-91. 
West, S.G., Hecker, K.D., Mustad, V.A., Nicholson, S., Schoemer, S.L., Wagner, P., et al. 2005. Acute effects of monounsaturated fatty acids with and without omega-3 fatty acids on vascular reactivity in individuals with type 2 diabetes. Diabetologia 48(1): 113-22.

Witte, D.R.W.J., de Konin EJ, van der Graaf Y, Grobbee DE, Bots ML 2005. Is the association between flow-mediated vasodilation and cardiovascular risk is limited to low-risk population? J Am Coll Cardiol 45(12): 1987-93.

Wopereis, S., Wolvers, D., van Erk, M., Gribnau, M., Kremer, B., van Dorsten, F.A., et al. 2013. Assessment of inflammatory resilience in healthy subjects using dietary lipid and glucose challenges. BMC Med Genomics 644.

Zilversmit, D.B. 1979. Atherogenesis: a postprandial phenomenon. Circulation 60(3): 47385. 


\section{TABLES}

Table 1. Nutritional composition of both test meals

\begin{tabular}{ccc}
\hline & HSFAM & MMM \\
\hline Total calories (kcal) & 858 & 885 \\
Total lipids (g) & 56.91 & 51.30 \\
Cholesterol (mg) & 252 & 76 \\
Total calories from fat (\%) & 58 & 51 \\
SFA (g) & 14.78 & 7.87 \\
MUFA (g) & 22.62 & 25.94 \\
Total PUFA (g) & 11.15 & 8.59 \\
Omega-3 PUFA (g) & - & 2.29 \\
DHA:EPA & - & $2: 1$ \\
Carbohydrates (g) & 68.74 & 63.81 \\
Fibers (g) & 6.56 & 13.00 \\
Proteins (g) & 24.16 & 37.82 \\
Sodium (mg) & 1806 & 1284 \\
Vitamin C (mg) & 4.77 & 87.93 \\
\hline
\end{tabular}

DHA: Docosahexaenoic acid, EPA: Eicosapentaenoic acid, MUFA: Monounsaturated fatty acid, PUFA: Polyunsaturated fatty acid and SFA Saturated fatty acid 
Table 2. Averaged baseline characteristics of study participants for both study visits.

\begin{tabular}{|c|c|c|c|}
\hline & All subjects & Group 1 & Group 2 \\
\hline $\mathrm{n}$ & 28 & 11 & 17 \\
\hline Age (years) & $33.8 \pm 8.3$ & $35.5 \pm 7.0$ & $32.7 \pm 9.0$ \\
\hline BMI $\left(\mathrm{kg} / \mathrm{m}^{2}\right)$ & $25.2 \pm 3.1$ & $26.9 \pm 3.1^{*}$ & $24.1 \pm 2.5$ \\
\hline Total-body fat (\%) & $18.3 \pm 6.3$ & $21.3 \pm 4.0$ & $16.6 \pm 6.8$ \\
\hline Trunk fat mass (\%) & $20.5 \pm 7.3$ & $23.5 \pm 4.7$ & $18.4 \pm 8.1$ \\
\hline Glucose $(\mathrm{mmol} / \mathrm{L})$ & $4.8 \pm 0.4$ & $4.9 \pm 0.3$ & $4.7 \pm 0.3$ \\
\hline Insulin (pmol/L) & $38[28,55]$ & $62[53,86] \dagger$ & $29[28,35]$ \\
\hline HOMA-IR & $1.0[0.8,1.8]$ & $2.0[1.7,2.8] \dagger$ & $0.8[0.7,1.0]$ \\
\hline Total cholesterol (mmol/L) & $4.5 \pm 1.0$ & $5.1 \pm 1.0$ & $4.2 \pm 0.7$ \\
\hline LDL (calculated) (mmol/L) & $2.8 \pm 0.9$ & $3.3 \pm 0.9 \dagger$ & $2.5 \pm 0.8$ \\
\hline $\mathrm{HDL}(\mathrm{mmol} / \mathrm{L})$ & $1.3 \pm 0.4$ & $1.1 \pm 0.2 \dagger$ & $1.5 \pm 0.4$ \\
\hline Non-HDL (mmol/L) & $3.2 \pm 1.1$ & $4.0 \pm 1.0 \dagger$ & $2.8 \pm 0.8$ \\
\hline Triglycerides $(\mathrm{mmol} / \mathrm{L})$ & $0.8[0.7,1.3]$ & $1.4[1.1,1.8] \dagger$ & $0.7[0.6,0.8]$ \\
\hline $\mathrm{C} / \mathrm{HDL}$ & $1.4 \pm 0.9$ & $5.0 \pm 1.2 \dagger$ & $3.1 \pm 1.0$ \\
\hline TG/HDL & $0.6[0.5,1.4]$ & $1.6[0.9,1.9] \dagger$ & $0.5[0.4,0.6]$ \\
\hline hsCRP (mg/L) & $0.8[0.3,0.9]$ & $0.8[0.6,1.0]$ & $0.8[0.2,0.8]$ \\
\hline FMD (\%) & $10.7 \pm 2.8$ & $11.0 \pm 3.6$ & $10.5 \pm 2.3$ \\
\hline Resting diameter (mm) & $3.8 \pm 0.4$ & $3.9 \pm 0.3$ & $3.7 \pm 0.4$ \\
\hline NMD (\%) & $11.8 \pm 3.1$ & $12.1 \pm 3.3$ & $11.5 \pm 3.0$ \\
\hline
\end{tabular}

BMI: Body-mass index, FA: Fatty acids, FMD: Flow-mediated dilatation, HDL: High-density lipoprotein, HOMA-IR: Homeostatic model assessment of insulin resistance, hsCRP: highsensitivity C-reactive protein, LDL: Low-density lipoprotein, NMD: Nitroglycerin-mediated dilatation and TG: Triglycerides 
Table 3. Scores of food frequency questionnaire evaluating dietary habits and habitual physical activity questionnaire evaluating fitness level

\begin{tabular}{cccc}
\hline & $\begin{array}{c}\text { All subjects } \\
(\mathrm{n}=28)\end{array}$ & $\begin{array}{c}\text { Group 1 } \\
(\mathrm{n}=11)\end{array}$ & $\begin{array}{c}\text { Group 2 } \\
(\mathrm{n}=17)\end{array}$ \\
\hline Global dietary score & $8.7 \pm 4.3$ & $8.3 \pm 4.1$ & $9.1 \pm 4.6$ \\
SFA score & $3.0 \pm 1.5$ & $3.1 \pm 1.6$ & $2.9 \pm 1.5$ \\
MUFA score & $2.0[1.0,4.0]$ & $2.0[1.5,3.0]$ & $2.5[1.0,4.0]$ \\
Omega-3 PUFA score & $4.0[2.0,8.0]$ & $4.0[3.5,8.0]$ & $3.5[2.0,8.0]$ \\
Fruit and vegetables score & $3.0 \pm 1.5$ & $3.9 \pm 1.4^{*}$ & $5.1 \pm 1.7$ \\
Omega-6 : omega-3 PUFA & $3.6[2.7,4.3]$ & $3.4[2.9,4.0]$ & $3.7[2.4,4.5]$ \\
& & $49.0[42.8$, & $51.5[46.3$, \\
Estimated $\mathrm{VO}_{2}$ max (ml / (kg x min)) & $49.7[45.2,55.8]$ & $51.2]$ & $55.8]$
\end{tabular}

* p-value < 0.05 vs. Group 2.

MUFA: Monounsaturated fatty acid, PUFA: Polyunsaturated fatty acid and SFA Saturated fatty acid 


\section{FIGURE LEGENDS}

Figure 1. Metabolic response to meals. Postprandial insulin $(A)$ and triglyceride $(B)$ variations (expressed in percentage of variation relative to baseline) to test meal ingestions.

* Wilcoxon signed-rank p-value $<0.05$, † Wilcoxon signed-rank p-value $<0.01$, Mann Whitney p-value $<0.05$

HSFAM: High-saturated fatty acid meal and MMM: Mixed Mediterranean-type meal

Figure 2. Endothelial function. Absolute endothelial function variations 4 hours following meal ingestion for both subgroups (expressed in \%FMD units variation from baseline).

* Student $t$-test $\mathrm{p}$-value $<0.01+$ Student $t$-test $\mathrm{p}$-value $<0.005$

FMD: Flow-mediated dilatation, HSFAM: High-saturated fatty acid meal and MMM: Mixed Mediterranean-type meal

Figure 3. Plasma total fatty acid profile. Fasted and postprandial plasma fatty acid composition. Values represent the proportion of each fatty acid with respect to total plasma fatty acid content, free and bound to triglycerides and phospholipids. Omega- 6 and omega- 3 PUFA values have been reproduced with a larger scale (in \%Total FA) for ease of visualization.

* Wilcoxon P-value < 0.05 vs. baseline and † vs. HSFAM

HSFAM: High-saturated fatty acid meal and MMM: Mixed Mediterranean-type meal 
A.

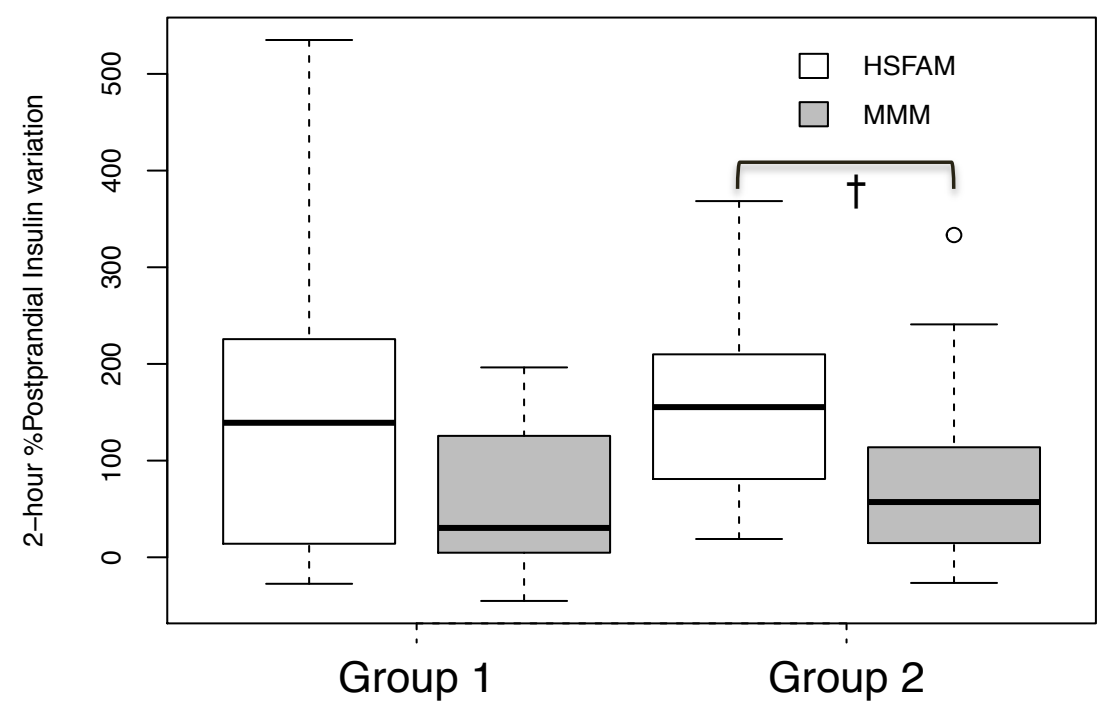

B.

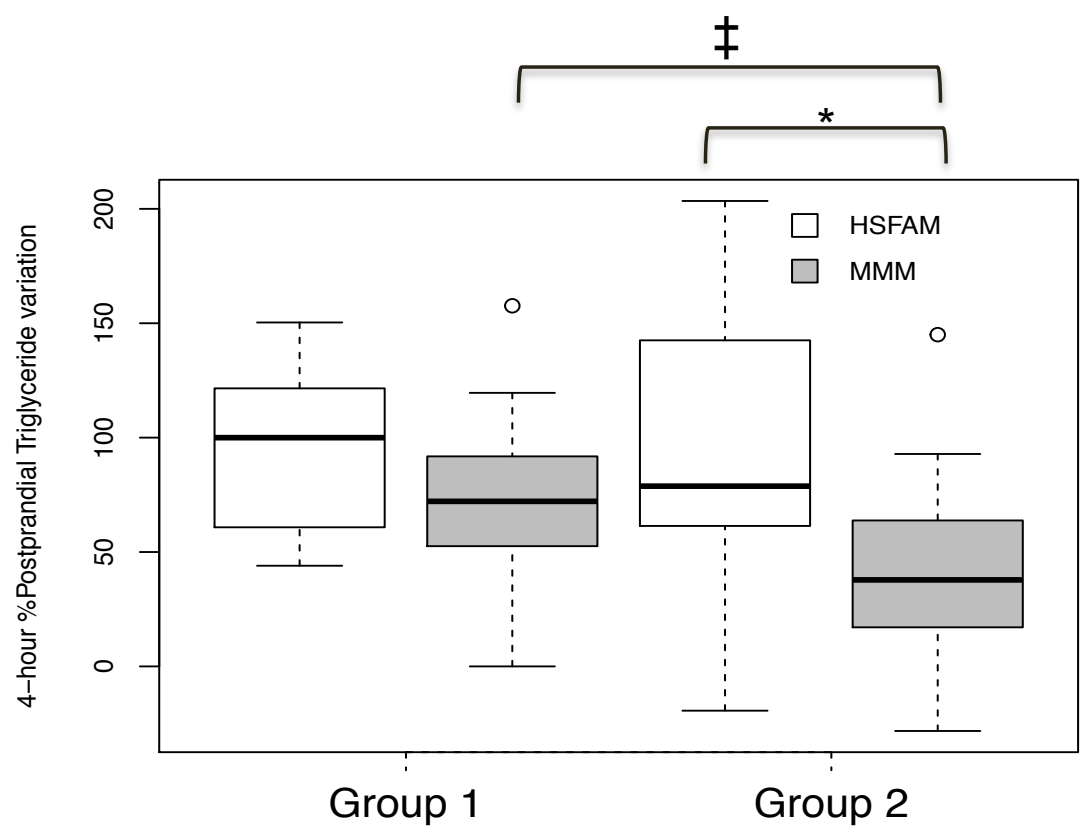


Figure 2.

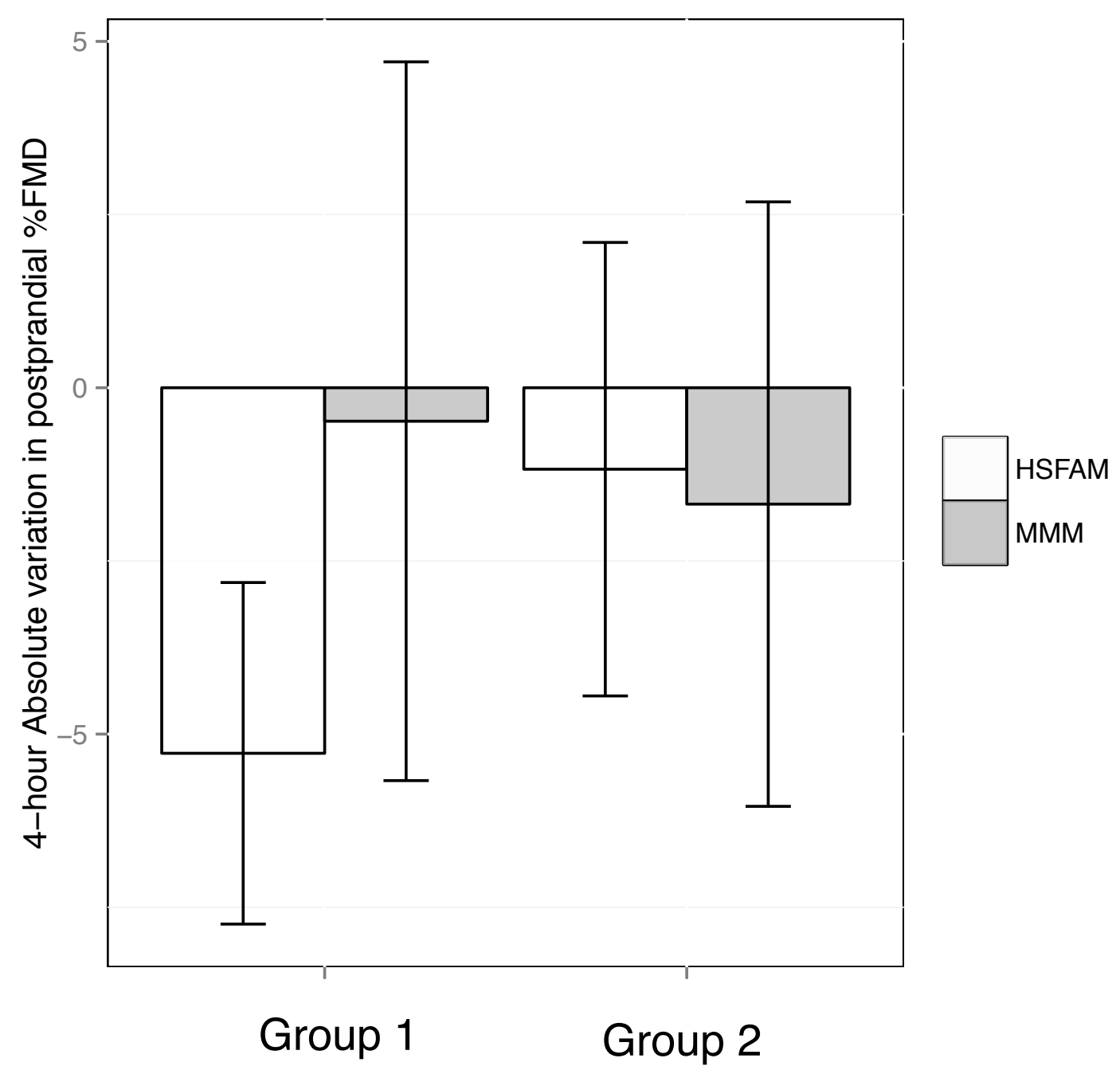

https://mc06.manuscriptcentral.com/apnm-pubs 
Figure 3.

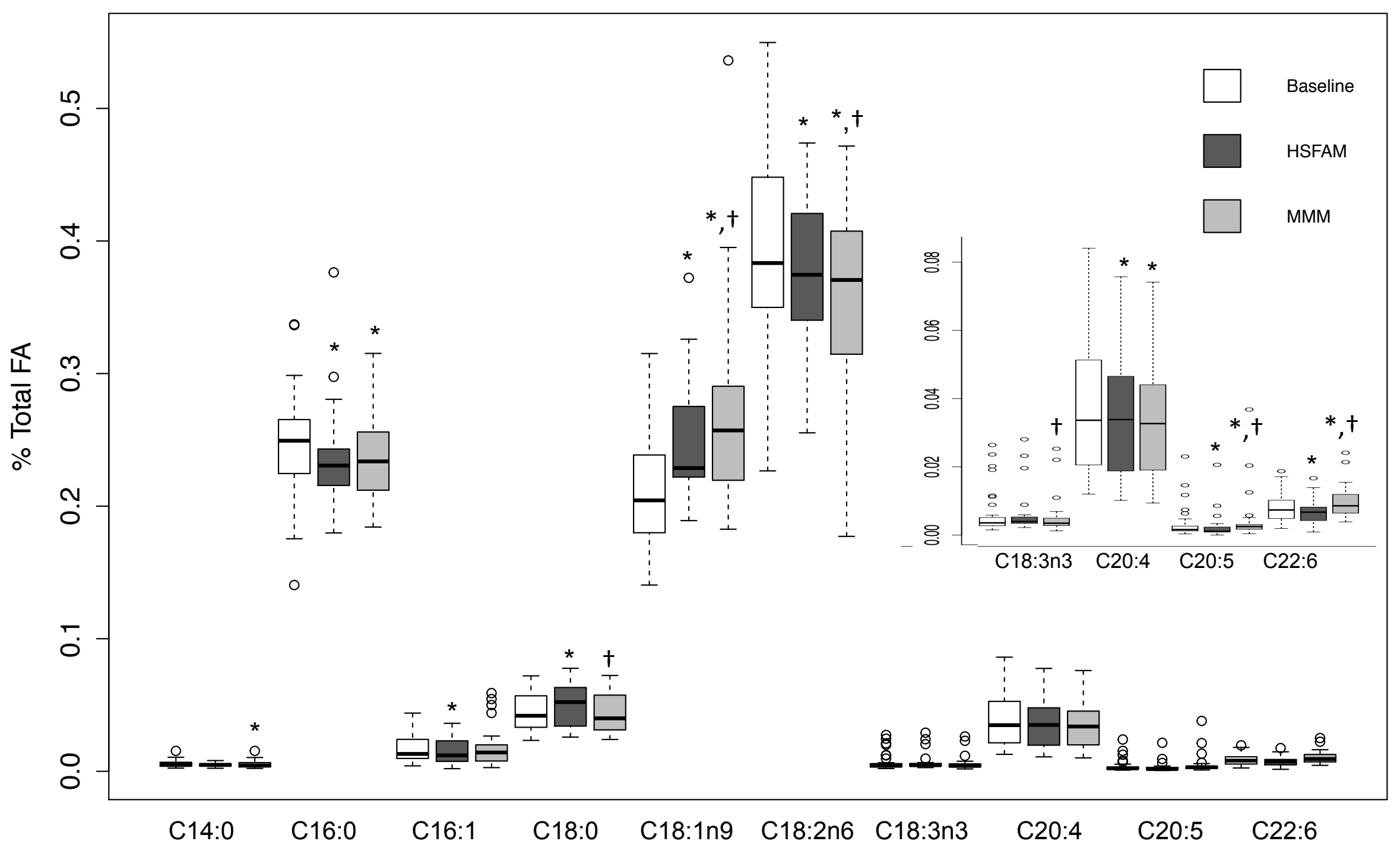

https://mc06.manuscriptcentral.com/apnm-pubs 